\title{
Building blocks for commodity augmented reality-based molecular visualization and modeling in web browsers
}

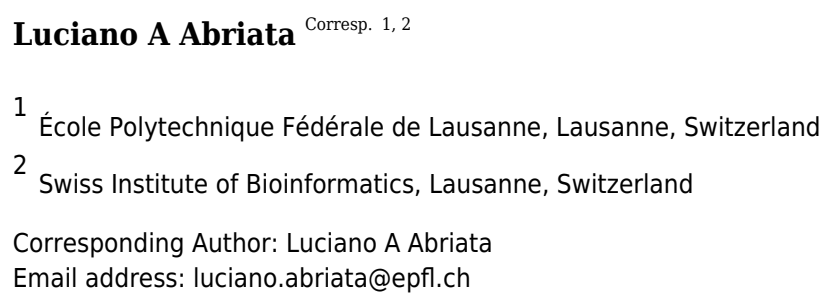

For years, immersive interfaces using virtual and augmented reality (AR) for molecular visualization and modeling have promised a revolution in the way how we teach, learn, communicate and work in chemistry, structural biology and related areas. However, most tools available today for immersive modeling require specialized hardware and software, and are costly and cumbersome to setup. These limitations prevent wide use of immersive technologies in education and research centers in a standardized form, which in turn prevents large-scale testing of the actual effects of such technologies on learning and thinking processes. Here, I discuss building blocks for creating marker-based AR applications that run as web pages on regular computers, and explore how they can be exploited to develop web content for handling virtual molecular systems in commodity AR with no more than a webcam- and internet-enabled computer. Examples span from displaying molecules, electron microscopy maps and molecular orbitals with minimal amounts of HTML code, to incorporation of molecular mechanics, real-time estimation of experimental observables and other interactive resources using JavaScript. These web apps provide virtual alternatives to physical, plastic-made molecular modeling kits, where the computer augments the experience with information about spatial interactions, reactivity, energetics, etc. The ideas and prototypes introduced here should serve as starting points for building active content that everybody can utilize online at minimal cost, providing novel interactive pedagogic material in such an open way that it could enable mass-testing of the effect of immersive technologies on chemistry education. 
1 Building blocks for commodity augmented reality-

2 based molecular visualization and modeling in web

3 browsers

4

Luciano Andrés Abriata ${ }^{1}$

${ }^{1}$ École Polytechnique Fédérale de Lausanne and Swiss Institute of Bioinformatics, Lausanne, Switzerland.

Corresponding Author: Luciano A. Abriata ${ }^{1}$

AAB044 Station 19, CH-1015 Lausanne, Switzerland

Email address: luciano.abriata@epfl.ch

\section{Abstract}

For years, immersive interfaces using virtual and augmented reality (AR) for molecular visualization and modeling have promised a revolution in the way how we teach, learn, communicate and work in chemistry, structural biology and related areas. However, most tools available today for immersive modeling require specialized hardware and software, and are costly and cumbersome to setup. These limitations prevent wide use of immersive technologies in education and research centers in a standardized form, which in turn prevents large-scale testing of the actual effects of such technologies on learning and thinking processes. Here, I discuss building blocks for creating marker-based AR applications that run as web pages on regular computers, and explore how they can be exploited to develop web content for handling virtual molecular systems in commodity AR with no more than a webcam- and internet-enabled computer. Examples span from displaying molecules, electron microscopy maps and molecular orbitals with minimal amounts of HTML code, to incorporation of molecular mechanics, real-time estimation of experimental observables and other interactive resources using JavaScript. These web apps provide virtual alternatives to physical, plastic-made molecular modeling kits, where the computer augments the experience with information about spatial interactions, reactivity, energetics, etc. The ideas and prototypes introduced here should serve as starting points for building active content that everybody can utilize online at minimal cost, providing novel interactive pedagogic material in such an open way that it could enable mass-testing of the effect of immersive technologies on chemistry education.

\section{Introduction}

For a long time it has been suggested that visual immersive analytics based in virtual reality (VR), augmented reality (AR) and other advanced forms of human-computer interactions have enormous potential in assisting thinking processes in scientific research and in education, especially in areas 
40 of science that deal with abstract objects, objects much smaller or larger than human dimensions, 41 objects that are hard to acquire and handle due to high costs, scarcity or fragility, and very large

amounts of data (O’Donoghue et al., 2010; Matthews, 2018; M. Krichenbauer et al., 2018; Sommer et al., 2018). Chemistry and structural biology are examples of such disciplines where AR and VR have been attributed high potential in education and research, by providing hybrid physical/computational interfaces to handle and explore virtual molecules in real 3D space augmented with real-time overlay of information from databases and calculations. However, the actual impact of immersive technologies on teaching, learning and working in chemistry still requires deep evaluation (M. Fjeld \& B. M. Voegtli, 2002; Pence, Williams \& Belford, 2015; Matthews, 2018; Bach et al., 2018; Yang, Mei \& Yue, 2018). Such evaluation has progressed very slowly due to the complex software setups and the specialized hardware needed, which limit availability, reach, adoption, and thus testing. Indeed this limitation is shared more broadly with other potential applications of AR and VR in science, which so far "[...] remain niche tools for scientific research" (Matthews, 2018).

In the last decade several groups have been studying ways to achieve immersive environments for chemistry and structural biology using VR and AR (Gillet et al., 2004, 2005; Maier, Tönnis \& GudrunKlinker, 2009; Maier \& Klinker, 2013; Hirst, Glowacki \& Baaden, 2014; Berry \& Board, 2014; Martínez-Hung, García-López \& Escalona-Arranz, 2016; Vega Garzón, Magrini \& Galembeck, 2017; Balo, Wang \& Ernst, 2017; Goddard et al., 2018b; Wolle, Müller \& Rauh, 2018; O’Connor et al., 2018; Ratamero et al., 2018; Müller et al., 2018; Goddard et al., 2018a; Stone, 2019). Such interfaces allow handling molecules over 6 degrees of freedom and with both hands, in immersive 3D. They are expected to overcome the limitations of traditional software based on screen, mouse and keyboard, thus enabling more intuitive, fluid exploration of molecular features and data. At the time of writing, most of these works are not complete AR or VR versions of fully-fledged programs, but rather prototypes, proofs of concept and case studies on how humans interact with virtual molecules in AR or VR. Notable highlights moving towards complete immersive molecular visualization and modeling programs are the new rewrite of Chimera, ChimeraX, which was optimized for the new GPUs and incorporates support for VR experiences (Goddard et al., 2018b), VRmol (Xu et al., 2019), new VR plugins for the VMD molecular graphics program (Stone, 2019), and a few commercial programs like Autodesk's 
70 Molecule Viewer (https://autodeskresearch.com/groups/lifesciences) or Nanome

71 (https://nanome.ai/), all with interfaces specifically tailored for VR.

72 Most of these works suffer from two severe limitations. First, all but a few exceptions

73 require hardware such as head-mount displays (helmets, headsets or goggles like MS Hololens,

74 Oculus Rift, HTC Vibe, etc.) or immersive installations with large surround screens plus 3D-

75 handheld input devices and the corresponding computer and GPU hardware. The few remarkable

76 exceptions are prototypes using ordinary webcam-enabled smartphones (Balo, Wang \& Ernst,

77 2017) or laptops (Gillet et al., 2004). The second limitation is that such specialized hardware

78 requires specialized programs that often must be correctly interfaced to couple the different

79 components required for an AR or VR experience, i.e. tracking limbs, handheld devices or AR

80 markers, then running calculations on molecular data, and finally displaying results and molecular

81 graphics on screen (for examples see (Ratamero et al., 2018) and (Gillet et al., 2005)). Some of

82 these programs are only compatible with specific VR devices and many are not free software.

83 Overall, then, despite the dropping costs, access to these tools still requires investment in the order

84 of hundreds to low-thousand American dollars per user, and software interfacing that may not be

85 available to lay students and teachers. It is therefore unlikely VR will achieve the ideal of "one

86 device per student" within the next few years. To date, these solutions are not widely used across

87 the world, and their costs make them totally out of reach for educational centers in developing

88 countries. Additionally, most current setups are limited to VR, but it has been shown that AR is

89 more suited for educational purposes because by not occluding the view of the user's own limbs,

90 it results in better motion coordination and object handling than VR (Sekhavat \& Zarei, 2016; Y.

91 Gaffary et al., 2017; M. Krichenbauer et al., 2018). Furthermore, in AR the view of the world is

92 not obstructed thus allowing students and teachers to interact more easily.

93 The purpose of this work is to demonstrate that client-side web technologies have matured

94 enough to enable web pages for AR-based molecular visualization and modeling running just on

95 web browsers in regular webcam-equipped computers. This enables the easy creation of immersive

96 educational material that can be employed by students and teachers at very affordable costs and

97 with very simple setups. All they must do is access a webpage, enable webcam activity in the

98 browser, and show to the webcam a printed AR marker on which the molecules will be displayed.

99 From the developer side, the code is made simple thanks to a large number of libraries; in fact 
100 visualization-only applications are achievable just with HTML code while interactivity can be 101 incorporated through custom JavaScript.

102 This article is organized in two parts. Part 1 provides a practical overview of the main 103 building blocks available as of 2019 to program AR apps in web pages, with a focus on ways to 104 achieve molecular visualization and modeling. It also briefly explores ways to handle gesture- and 105 speech-based commands, molecular mechanics, calculation of experimental observables, 106 concurrent collaboration through the world wide web, and other human-computer interaction 107 technologies available in web browsers. Part 2 of the article showcases prototype web apps for 108 specific tasks of practical utility in pedagogical and research settings. These web apps are based 109 on open, commodity technology that requires only a modern browser "out of the box", so 110 educators, students and researchers are free to try out all these examples on their computers right 111 away by following the provided links.

112

Part 1: Overview of building blocks for immersive molecular modeling in web browsers

\section{Virtual and augmented reality}

At the core of immersive experiences are visualizations based on virtual or augmented reality methods. While VR is probably best experienced with VR goggles to suppress any side view of the real world, AR is more amenable to devices like desktop or laptop computers, tablets and smartphones, making it better suited for commodity solutions, and has the additional advantages outlined in the introduction. The methods presented in this article make use of markerbased AR in a web-based setup that functions as an "augmented reality mirror" where the user sees him/herself with the virtual objects, here molecules, overlaid on the markers he/she holds in their hands (Figure 1A,B). The following descriptions focus on this technology and how it can be implemented in web apps by using HTML, CSS and JavaScript code as standard web pages that are hosted at servers but run entirely on local computers (Figure 1C).

The WebGL API provides powerful 2D and 3D graphing capabilities using GPU resources, in a format fully integrable with other HTML elements, APIs and JavaScript libraries, without the need of plug-ins; and is highly standardized across browsers. It is thus possible to couple all elements required to build up an AR experience directly inside the web page source code. A handful of JavaScript libraries exploit WebGL to facilitate rendering of 3D scenes, Three.js 
131 (https://threejs.org/) being probably the most widely used. In turn, tools like A-Frame 132 (https://aframe.io/) provide entity component system frameworks that wrap Three.js into HTML 133 language tags through polyfills, greatly facilitating the development of AR and VR scenes. The 134 examples presented in Part 2 showcase either direct use of Three.js or of Three.js through A-Frame 135 for AR. These libraries/frameworks can be used either by (i) loading pre-made models of the 136 molecular systems in formats like Wavefront's OBJ+MTL files exported straight out of molecular 137 visualization programs like VMD (Humphrey, Dalke \& Schulten, 1996), UnityMol (S. 138 Doutreligne et al., 2014; Wiebrands et al., 2018), ChimeraX (Goddard et al., 2018b), etc., possibly 139 further edited with a program like Blender as in (Martínez-Hung, García-López \& Escalona140 Arranz, 2016)); or (ii) employing 3D primitives (like spheres, cylinders, etc.) to draw the molecular 141 systems from scratch with their atomic coordinates. Use of Wavefront models is much simpler 142 (only a few lines of HTML code to load and display objects) and allows seamless display of any 143 element exported from molecular graphics programs, including not only different representations 144 of molecules but also isosurfaces as those needed to visualize volumetric data describing molecular 145 orbitals, electron microscopy maps, etc. On the other hand, using 3D primitives requires larger 146 pieces of code to create all 3D objects from atomic coordinates, but in turn allows for fine dynamic 147 control of shapes, sizes, colors, and positions, which are key to interactivity. Another downside of 148 Wavefront models is that files can become quite large for high-resolution textures.

\section{Object detection and tracking}

151 The other key component required for AR and VR is a means to detect and track objects or parts 152 of the user's body such as his/her hands, in order to manipulate virtual objects. Applications using ad hoc hardware use sensors and cameras that track the user's position in space and handheld controllers that the user sees as virtual tweezers or hands, to directly move objects in space. For commodity AR/VR in web browsers, solutions rely on JavaScript versions of tracking libraries that implement computer vision algorithms through the webcam feed, like ARToolKit (Kato, 1999) among other similar solutions. These libraries track user-defined 2D markers (like those in Figure 1A, Figure S1, Figure S2) in space and make their computed coordinates available to the graphics algorithms. Two especially interesting libraries, used in many of the examples presented in Part 2, are AR.js (https://github.com/jeromeetienne/AR.js) and its A-Frame wrap 
161 (https://jeromeetienne.github.io/AR.js/aframe/). These enable highly simplified AR/VR, even 162 using exclusively HTML code for simple developments.

163 It is important to note that in marker-based AR different viewers looking at the same 164 physical marker receive different perspectives of it and hence of the rendered virtual object, just 165 as if it was a real object in real space (Figure S3). This easily enables multi-user AR in a common 166 room, as would be useful in a classroom setting where students and teachers look at the same 167 virtual molecule.

An alternative to traditional marker-based AR should in principle be possible by using a 169 plain hand tracking JavaScript library like Handtracking.js. Another slightly more expensive approach but possibly better in tracking performance is using a device like the infrared-based Leap Motion Controller (https://www.leapmotion.com/), which includes a JavaScript library to feed positional information from the device into the web app. Unfortunately, however, there are currently no ready-to-use libraries that couple these input tools to WebGL graphics, so their use would require further developments.

Current JavaScript libraries for computer vision allow even more complex object tracking. One interesting example is gesture recognition by the WebGazer.js library, which analyzes face features to estimate where on the screen the user is looking at (Papoutsaki et al., 2016). In molecular visualization, this can be used for example to automatically rotate regions of interest to the front of the field of view, as shown in Figure S4.

\section{Speech-based interfaces}

A speech-based interface can be very useful for situations in which the user's hands are busy holding objects, as in AR/VR applications. In-browser speech recognition APIs enable implementation of speech recognition very easily, especially through libraries like Annyang (Ater, 2019) which is used in some of the examples of this article. These libraries usually allow working in two modes, one where the browser waits for specific commands (while accepting variable arguments) and one where the browser collects large amounts of free text that are then made available to the environment. The former allows direct activation of functions without the need for the user to click on the screen. The second option opens up the possibility of automatically detecting subjects, actions and concepts that are fed to artificial intelligence, or just predefined 
192 the interaction surface between two proteins and mention certain residues, the computer could 193 automatically mine NIH's PubMed repository of papers for mentions of said residues. This may 194 seem far-fetched, but is essentially the same technology that underlies automatic advertising and 195 suggestions based on users' various inputs and usage statistics in software and websites. The 196 problem of intelligently suggesting chemical and biological information related to a topic or object 197 has already been addressed for some time, for example in information augmentation tools like 198 Reflect (Pafilis et al., 2009) and advanced text-mining tools (Rebholz-Schuhmann, Oellrich \& 199 Hoehndorf, 2012). The evolution of science-related standards are very important in this regard, 200 formats and content for the semantic web (Hendler, 2003) and of machine-readable scientific 201 databases and ontologies that standardize knowledge and data.

202

203 Intensive calculations

204 As recently reviewed in a dedicated issue of Computing and Science in Engineering (M. DiPierro, 205 2018), JavaScript has become very powerful by including language subsets specialized for speed, 206 optimized just-in-time compilation, methods to program background scripts, and libraries to 207 perform multi-core and on-GPUs computing to accelerate intensive calculations. It is in fact now 208 possible to transpile from $\mathrm{C} / \mathrm{C}++$ and other languages directly to JavaScript retaining close-tonative execution speeds, allowing web browsers to support quite complex data analysis and visualization directly in web pages (Abriata et al., 2017; Abriata, 2017a). This opens up the possibility of simulating molecular mechanics and experimental data, performing numerical data analysis and even handling data in neural networks, directly inside the molecular modeling web app to enable real-time feedback as the user manipulates the molecular systems. Some of the prototypes presented in Part 2 include such examples.

Rather than manually implementing calculations, a number of libraries are now available

217 being developed by specialists (Abriata, 2017a). One particularly useful library in the scope of this 218 paper is Cannon.js (https://schteppe.github.io/cannon.js/), an engine for simulating rigid body 219 mechanics that integrates with Three.js and A-Frame. These engines use numerical methods to 220 propagate motions of solid bodies connected by springs and other physical constraints in an 221 approximate but efficient way, thus adding realistic physics to the 3D bodies of AR and VR worlds. Although these engines do not account for all the forces and phenomena of the atomic realm, such 
223 as electrostatic interactions and quantum phenomena, they are useful in certain real scenarios of 224 molecular modeling. For example, the Integrative Modeling Platform software (used to put 225 together partial structures into bigger assemblies, driven by experimental data) includes one such 226 kind of physics engine for initial molecule-molecule docking (Russel et al., 2012). Furthermore, 227 implementation of more complex force fields is certainly possible, as exemplified by a JavaScript 228 transpilation of the OpenMD molecular dynamics engine (Jiang \& Jin, 2017).

229

\section{Further building blocks}

231

232

233

234

235

236

237

238

239

240

241

242

243

244

245

246

247

248

249

250

251

252

253

Any other technology that facilitates interaction with the computer within a 3D environment, either to deliver or obtain information, might be of use. For example, haptic feedback would be desirable to confer a physical feel of interactions and clashes as in (Wollacott \& Merz, 2007; Stocks, Hayward \& Laycock, 2009; Stocks, Laycock \& Hayward, 2011; Matthews et al., 2019). Achieving a good experience in haptic feedback currently requires specialized devices, and is an area of active research (Bolopion et al., 2009, 2010), therefore it does not fit with the commodity hardware criteria outlined in the introduction. Other rudimentary ways to achieve sensory feedback include exploiting built-in vibration devices and touch-pressure sensing in touch screens, both handled by JavaScript APIs.

Finally, a particularly interesting aspect of software running on web browsers is the ease with which different users can connect to each other, just over the internet. Web apps can exploit web sockets to achieve direct browser-to-browser links over which data can be transmitted freely, with a server only intervening to establish the initial connection (V. Pimentel \& B. G. Nickerson, 2012). For example, two or more users can concurrently work on a JSmol session by sharing just mouse rotations and commands, appearing on all other users' screens with a minor delay (Abriata, 2017b). Such collaborative working technology could be adapted to complex immersive environments to allow multiple users to work on chemical problems at a distance, essential for scientific collaborations, demonstrations, and online teaching (Lee, Kim \& Kang, 2012).

\section{Part 2: Prototype web apps showcasing example applications}

This section presents example AR web apps compatible with major web browsers in modern computers, introducing features of increasing complexity. All examples were verified to run out of the box in multiple web browsers on Windows, Linux and MacOS operating systems, in laptop 
254 and desktop computers. All the examples are accessible through links in Table 1 and at 255 https://lucianoabriata.altervista.org/papersdata/tablepeerjcs2019.html, which further contains 256 links to demo videos. To run these examples the user needs to print the Hiro, Kanji or cube markers 257 as needed in each case (Figure 1A, Figure S1, Figure S2, and links on web pages). For simpler 258 handling, flat markers (Hiro and Kanji) may be glued on a flat surface mounted on a device that 259 can be easily rotated from the back, such as a small shaft perpendicular to the marker plane. The

260

261

262

263

264

265

266

267

268

269

270

271

272

273

274

275

276

277

278

279

280

281

282

283

284 cube marker is printed in a single page, folded and possibly glued to a solid cube made of wood, plastic, rubber or similar material. Readers interested in the inner workings and in developing content can inspect the source code of each webpage ( $\mathrm{Ctrl}+\mathrm{U}$ or $\mathrm{Cmd}+\mathrm{U}$ in most browsers). Several recommendations and basic troubleshooting for developers and users are given in Table 2 .

\section{Introducing web browser-based AR for visualization}

The simplest way to achieve AR in web pages consists in displaying on the AR markers representations exported from programs like VMD (Humphrey, Dalke \& Schulten, 1996) in Wavefront (OBJ+MTL) format. This can be achieved with a few lines of HTML code thanks to libraries like AR.js for A-Frame, enabling the very easy creation of content for displaying any kind of object handled by the exporting program. Figure 2A exemplifies this with a small molecule, 2bromo-butane, shown as balls and sticks. This small molecule is chiral at carbon 2; the Hiro marker displays its $\mathrm{R}$ enantiomer while the Kanji marker displays the $\mathrm{S}$ enantiomer, both rendered from the same pair of OBJ+MTL files but scaled as required for chirality inversion. Figure 2B shows on the Hiro marker a protein complex rendered as cartoons with small molecule ligands rendered as sticks (PDB ID 1VYQ, same example used by (Berry \& Board, 2014)); and Figure 3 shows a cartoon representation of a protein bound to a short segment of double stranded DNA rendered as sticks (from PDB ID 1FJL) spinning on the Kanji marker. Figure 2D exemplifies display of VMD isosurfaces to show a volumetric map of a bacteriophage attached to its host as determined through electron microscopy (EMDB ID 9010). Two further examples feature combined representations of atomic structure and volumetric data: Figure 2E shows a small peptide rendered as sticks and docked inside the experimental electron map shown as a mesh (from PDB ID 3HYD), and Figure $2 \mathrm{~F}$ shows the frontier molecular orbitals of $\mathrm{BH}_{3}$ and $\mathrm{NH}_{3}$ (Wavefront files kindly provided by $\mathrm{G}$. Frattini and Prof. D. Moreno, IQUIR, Argentina). 

advantage over ready-made models that all the elements can be handled independently, thus 287 allowing the possibility of incorporating interactivity. This can be achieved either through A288 Frame with AR.js, thus requiring only HTML code for display as in the Wavefront-based examples 289 above, or through libraries that require additional JavaScript code but in turn enable more complex 290 functionalities. Exemplifying the former case, Figure 2G uses A-Frame spheres and cylinders 291 placed at coordinates computed from atomic positions, colored by atom type and assigned atom292 specific radii, to render a model of a small protein helix on the Kanji marker. The latter case of 293 using pure JavaScript is illustrated by the jsartoolkit library 294 (https://github.com/artoolkit/jsartoolkit5), in an example where six markers arranged on the faces 295 of a cube are coupled to drive a single object in full 3D. Lastly, combining this with direct use of 296 Three.js to draw 3D primitives, the web app in Figure 2H allows visualization and manipulation 297 of any molecule loaded in PDB format in full 3D, in this case (D)-glucopyranose.

\section{Adding interactivity: examples on small molecules}

300 Web apps using A-Frame can gain interactivity through portions of JavaScript code that read atom 301 coordinates and carry out calculations on them. Figure 3 shows another series of examples of increasing complexity, focusing on small molecules. In Figure 3A, the user drives a lysine side chain with the Hiro marker and a glutamate side chain with the Kanji marker. Each molecule is anchored to the center of its corresponding AR marker through its alpha carbon atom. Their protonation states correspond to neutral $\mathrm{pH}$, so lysine is protonated hence its $\mathrm{N}$ atom (blue) is charged by +1 , whereas glutamate is deprotonated hence its $\mathrm{O}$ atoms (red) bear a total charge of connecting the lysine's $\mathrm{N}$ atom and one of the glutamate's $\mathrm{O}$ atoms; (ii) reports the distance between these atoms and the corresponding attractive electrostatic force in real time; and (iii) checks for and displays clashes between any pair of atoms of the two molecules. The code for (i)

311 is wrapped inside an auxiliary .js file, and the code for (ii) and (iii) is located between $<$ script $>$ 312 tags at the end of the HTML file. The distance, electrostatic and clash calculations are computed 313 inside a setInterval() function every $200 \mathrm{~ms}$, and use the id attribute of the sphere tags to locate the 314 atomic coordinates. The distance calculation includes a correction for a zoom factor that scales 315 atom sizes and positions when the molecular coordinates are parsed into A-Frame HTML to 
316 properly fit the screen. Clashes are detected when two spheres are within $3 \AA$ and displayed as 317 semitransparent A-Frame spheres centered on the affected atoms.

318 A modified version of this example is also provided, incorporating a very simplistic 319 emulation of hydrogen bond detection and proton transfer (second link for Figure 3A in Table 1). 320 JavaScript code calculates and displays hydrogen bonds when the geometry permits, and randomly 321 "transfers" one proton from lysine's $\mathrm{N}$ atom to one of the $\mathrm{O}$ atoms of glutamate if the lysine is 322 protonated, or the other way around (actually spheres attached to each marker are hidden or shown 323 as needed to emulate proton transfer). Protons "jump" only when they are within $2 \AA$ of the 324 receiving $\mathrm{N}$ or $\mathrm{O}$ atom; and they are set to jump back and forth to reflect $70 \%$ time-averaged 325 population of protonated lysine and $30 \%$ of protonated glutamate, to convey the feeling of different 326 acidic constants $\left(10^{\text {-pKa }}\right)$ favoring protonation of the base. When $2 \AA<\mathrm{N}-\mathrm{O}$ distance $<3 \AA$ the web 327 app displays a yellow dotted line that represents a hydrogen bond between the potential receiver 328 heavy atom and the involved proton.

Similar emulation strategies could be easily used to build "interactive animations" for 330 exploring chemical and physical phenomena of much pedagogical use, as in the PhET interactive simulations (Moore et al., 2014) but using AR to directly, almost tangibly, handle molecules. For example, the app shown in Figure 3B illustrates stereoselectivity in the Diels-Alder reaction in interactive 3D. This reaction occurs between a dienophile and a conjugated diene in a concerted fashion, such that the side of the diene where the initial approach occurs defines the 335 stereochemistry of the product. The web app in this example allows users to visualize this in $3 \mathrm{D}$ as they approach a molecule of 1,3-cyclohexadiene on the Hiro marker to a molecule of chloroethene on the Kanji marker. As the two pairs of reacting $\mathrm{C}$ atoms approach each other, the new bonds gain opacity until the product is formed. Additionally, the product formed in this reaction is by itself an interesting molecule to visualize and manipulate in $\mathrm{AR}$, because it contains two fused six-membered rings which are hard to understand in 2D.

It should be noted that the examples provided here emulating reactivity are merely pictorial 342 visualizations of the mechanisms, and not based on any kind of quantum calculations. Such 343 calculations are too slow to be incorporated into immersive experiences where energies need to be 344 computed on the fly. However, novel machine learning methods that approximate quantum 345 calculations through orders-of-magnitude faster computations (like in (Smith, Isayev \& Roitberg, 346 2017; Bartók et al., 2017; Paruzzo et al.)) could in the near future be coupled to AR/VR systems 
347 to interactively explore reactivity in real time. Obviously, such tools could be useful not only for

348 education but also research, for example to interactively test the effect of chemical substituents on 349 a reaction, estimate effects on spectroscopic observables, probe effects of structural changes on 350 molecular orbitals, etc. It is already possible to integrate AR/VR with a physics engine, to add 351 realistic mechanics to the simulation. The web app in Figure 2H uses Cannon.js to simulate thermal 352 motions and thus give a sense of dynamics to the visualized system. In this web app, Cannon.js 353 handles the multi-atom system by treating atoms as spheres of defined radii connected by fixed354 distance constraints and whose velocities are continuously updated to match the set temperature, 355 leading to rotations around bonds. However, extension of Cannon.js to include additional force field terms like dihedral angle terms and electrostatic interactions would be needed to achieve a more complete and realistic modeling experience.

\section{AR-based modeling of biological macromolecules}

360

361

362

363

364

365

366

367

368

369

370

371

372

373

374

375

376

377

Figures 2 and 3 already include examples for visualizing certain biological macromolecules, and the previous section introduced ways to incorporate interactivity into these web apps. This section digs deeper into the development of interactive content more relevant to education and research in structural biology, by exploring the incorporation of restraints, simple force fields and on-the-fly simulation of experimental observables for biological macromolecules.

The example in Figure 3C uses JavaScript to calculate paramagnetic effects on the nuclear magnetic resonance signal of a probe atom (black) attached to the Kanji AR marker, as it is moved around the heme group of metmyoglobin attached to the Hiro AR marker. By applying standard equations (Bertini, Turano \& Vila, 1993) on the atomic coordinates, this web app simulates the dipolar effects of the paramagnetic iron ion on the probe atom and displays the resulting contribution to the NMR spectrum using the Google Charts JavaScript library (https://developers.google.com/chart).

The web app shown in Figure 4A allows exploration of the interaction space of two proteins that are known to form a complex in solution, specifically ubiquitin (red trace) and a ubiquitininteracting motif (UIM, blue trace) taken from PDB ID 2D3G (Hirano et al., 2006). The web app simulates on-the-fly the small-angle X-ray scattering (SAXS) profiles expected from the relative arrangement of the two proteins, and displays them overlaid onto an experimental profile in real time as the user moves the proteins. This offers a way to interactively test compatibility of possible 
378 docking poses with the experimental data. Although it cannot compete with the extensive sampling 379 achievable with molecular simulations, such an interactive tool could be useful for preliminary 380 analysis of SAXS data before starting complex calculations or to assist interpretation of the results 381 of such calculations. For simplicity and speed, in this example the SAXS profile calculation is 382 based on the Debye formula iterated through pairs of residues instead of through all pairs of atoms 383 as the full equation requires (Debye, 1915); however, realistic SAXS profiles of actual utility in 384 modeling can be achieved with coarse graining strategies and proper parameterization of the 385 scattering centers (Stovgaard et al., 2010). This web app further includes a rudimentary residue386 387 grained force field (i.e. describing each amino acid with one backbone and one side chain bead) to detect clashes, and a predefined binding coordinate which upon activation brings the two molecules together. Activation of SAXS profile simulation, clash-detecting force field and binding 389 coordinate are controlled by voice commands, required because the user's hands are busy handing 390 the markers. This proceeds through the browser's cloud-based speech recognition API so does not consume much resources. The successful combination of all these different elements (AR, 3D visualization, calculations, and speech recognition) illustrates the superb integration capability of libraries for client-side scripting in web browsers. The modularity and simplicity of client-side web programming allows easy adaptation to other kinds of experimental data; for example to residue-specific paramagnetic relaxation enhancements as done by Prof. Rasia (IBR-CONICETUNR, Argentina) at https://rrasia.altervista.org/HYL1_1-2/Hyl1_12_minima.html.

Another example, presented in Figure 4B shows how AR can help to explore residueresidue contact predictions from residue coevolution data. Such predictions provide useful restraints in modern approaches for modeling proteins and their complexes (Simkovic et al., 2017;

400

401

402

403

404 405 406 407 408 Abriata et al., 2018; Abriata, Tamò \& Dal Peraro, 2019), but often include a fraction of false positives that introduce incorrect information if undetected. Interactive inspection of residueresidue contact predictions could help to actively detect false positives through human intervention before the actual restraint-guided modeling proceeds. The example in Figure 4B shows contacts predicted from coevolution analysis of large sequence alignments for the pair of proteins in chains A and B of PDB ID 1QOP, taken from the Gremlin server (Ovchinnikov, Kamisetty \& Baker, 2014). Each protein is driven by one marker, and the predicted contacts are overlaid as dotted lines connecting the intervening pairs of residues. These lines are colored green, olive and red according to decreasing coevolution score as in the Gremlin website, and their widths reflect in real time the 
409 distance between pairs of residues, presumably minimal when contacts are satisfied if the 410 prediction is true.

411 The last prototype application shows rudimentary handling of highly disordered protein 412 regions, in this case to test how a flexible linker made of 6 glycine residues restricts the space 413 available for exploration and possible docking poses of two well-folded domains (Figure 5A-C). 414 Each folded domain (ubiquitin and ubiquitin-interacting motif, taken from PDB ID 2D3G) is 415 modeled at a resolution of 2 spheres per residue, one centered at the backbone's alpha carbon and 416 one at the center of mass of the sidechain (i.e. a description slightly coarser than that of the 417 MARTINI force field (Marrink et al., 2007)). All spheres representing residues of the folded 418 domains are kept in fixed positions relative to their AR marker, and have radii assigned as the 419 cubic root of the amino acid volumes to roughly respect the relative amino acid sizes (taken from 420 (Abriata, Palzkill \& Dal Peraro, 2015)). The glycine residues of the flexible linker are modeled as 421 single spheres centered at the alpha carbons with their radii set to the cubic root of glycine's 422 volume. Using Cannon.js, the spheres representing the glycine residues of the flexible linker (in 423 orange in Figure 5) are allowed to move freely but under a fixed-distance constraint from each

424 425 426

427 428 429 430 431 432

\section{Discussion}

434 Achieving seamless integration of immersive visualizations, haptic interfaces and chemical 435 436 437 438 439

other and from the corresponding ends of the folded domains. This very simple model can help to answer questions related to the separation of the anchor points and the maximal extension of the linker when straight: How far apart can the two proteins go with the given linker containing 6 residues? Can both proteins be docked through certain interfaces keeping the linker in a relaxed configuration? The user's investigations are assisted by on-the-fly estimation of entropy associated to given extensions of the linkers, estimated with a worm-like chain model from polymer physics (Marko \& Siggia, 1995), and by an estimation of the strain experienced by the linker when the user pulls its glycine residues apart beyond their equilibrium distance.

computation stands as one of the key "grand challenges" for the simulation of matter in the $21^{\text {st }}$ century (Aspuru-Guzik, Lindh \& Reiher, 2018). Such integration is expected to help us to more easily grasp and explore molecular properties and to efficiently navigate chemical information. In the last two decades several works introduced different ways to achieve AR and VR, as presented in the Introduction section. In education, such tools could replace or complement physical (such 
440 as plastic-made) modeling kits, augmenting them with additional information about forces, 441 charges, electron distributions, data facts, etc. In research, such tools could help better visualize 442 and probe molecular structure, simulate expected outcomes of experiments and test models and 443 simulated data against experimental data, etc., all through intuitive cues and fluent human444 computer interactions.

445 This article introduced a minimal set of building blocks and code for developing marker446 based AR applications for molecular modeling in web browsers using regular computers. Since 447 web AR is a new and emerging technology, it is reasonable to identify some limitations. Naturally, 448 such web apps cannot currently match the graphics quality and interfacing capabilities of programs 449 prepared for specialized AR/VR hardware, and the use of a webcam to follow markers can result 450 in unstable tracking under certain conditions compared to setups that use ad hoc devices. Another 451 limitation is that support for AR in smartphones is not uniform, and varies greatly between devices. 452 Currently, only laptops and desktop computers provide a consistent experience. Lastly, content 453 developers must routinely verify that the web apps continue to work in major browsers after 454 updates, and also validate them against new W3C standards, APIs and libraries. On the bright side, 455 these web apps have some important advantages. First, all examples presented here rely only on 456 client-side programming; therefore, applications need only be uploaded to regular webserver to 457 become available to the world. Second, they do not require client-side plugins, so are supported 458 "out of the box" by standard web browsers. Third, the modularity of JavaScript and the availability of several ready-to-use libraries greatly simplify development of new content. Fourth, users of these applications do not need to worry about updates, as they always receive the latest version when they reload the page. All in all, these positive features will favor adoption of browser based AR/VR for educational purposes over alternatives that require specialized software and hardware.

\section{Future Perspectives}

For educational applications, the next stage would be to develop actual content of value for 466 teachers and students. The simplest content could merely provide visualizations to explore molecular geometries, surfaces, orbitals, etc., with specific sets of demos to assist learning of key concepts such as chirality, organic molecules, metal coordination complexes and biomacromolecular structures, to mention just a few cases. By adding mechanics, more complex demos could be created where students could for example interactively explore torsion angles as 
471 in the textbook cases of probing the energy landscape of rotation around the central C-C bond of 472 butane, or swapping between chair and boat conformations of 6-member rings, exploring hydrogen 473 bonding patterns between neighbor beta strands in a protein, etc. Importantly, every single student 474 having a computer at hand could use these apps, not only at the school/university but also at home, 475 therefore this could become an actual learning tool of full-time, individual use. The possibility of 476 reaching the masses with this kind of web technologies for AR-based molecular modeling in turn 477 opens up the opportunity of performing large-scale evaluations of their actual impact in education. 478 As actively investigated by others, there is also a need to explore if full working programs 479 for AR-based molecular modeling may actually become powerful enough to also assist research. 480 Here again, web-based tools like those discussed in this article could help to carry out such tests 481 at large scales. Some of the prototypes presented here advance possible uses in research, as in the 482 simulation of data from protein-protein docking poses and comparison to the corresponding 483 experimental data in real time. However, some issues should be addressed before creating fully484 fledged web programs for research: (i) improving AR marker detection and tracking to stabilize 485 inputs (Q. H. Gao et al., 2017), (ii) developing some kind of AR marker that is clickable so that 486 users can drag and drop objects in space (hitherto unexplored), (iii) improving graphics, where the possibility of adapting existing web molecular graphics like NGL (Rose \& Hildebrand, 2015), 3dmol (Rego \& Koes, 2015), Litemol (Sehnal et al., 2017), Mol*(Sehnal et al., 2018), JSmol (Hanson et al., 2013), etc. is particularly enticing, and (iv) developing force fields that correctly handle molecular dynamics and energetics for different tasks, which may imply different levels of granularity for different applications. Another global improvement, also important for pedagogical applications, would be incorporating proper object occlusion, which is still non-trivial and subject of studies in the AR community (M. M. Shah, H. Arshad \& R. Sulaiman, 2012; J. Gimeno et al., 494 2018).

Some further directions that could be explored in the near future are fluently connecting through an AR experience users in physically distant locations, so that they can collaborate on a research project or teach/learn at a distance. Adapting future standards for commodity AR/VR in smartphones (plugged into cardboard goggles for stereoscopy) is also worth exploring as this would lead to an even more immersive experience than with the mirror-like apps proposed here. However, since smartphones are more limited in power, their use for AR molecular modeling may 
502 visual analytics for molecular modeling, and especially thinking about solutions useful for 503 research, a few especially enticing additions include support for haptic devices for force feedback, 504 AR without markers (i.e. just by tracking the users' hands and fingers) and considering occlusion, 505 and as described above, the capability to respond to visual or audio cues by automatically checking 506 databases and mining literature to propose relevant pieces of information.

507

\section{Acknowledgements}

509 I acknowledge all the members of the communities that develop the client-side web programming 510 tools used here, as well as the very helpful communities who contribute to their improvement, bug 511 detection and correction, documentation, and online help. Special thanks to J. Ethienne (AR.js 512 developer), Nicolò Carpignoli (AR.js maintainer), D. McCurdy (Google), T. Ater (Annyang 513 developer), L. Stemkoski (Adelphi U., USA), A. Herráez (U. Alcalá, Spain) and A. Papoutsaki 514 (Pomona College, USA) for assistance. I also acknowledge numerous researchers from the 515 physics, chemistry and biology communities who have provided ideas, suggestions, examples and 516 support, especially L. Krapp, S. Träger, M. Dal Peraro, F.G. van der Goot and M.E. Zaballa (EPFL, 517 Switzerland), A. Barducci (CBS, France), D. Moreno and G. Frattini (IQUIR-CONICET-UNR, 518 Argentina), and R. Rasia (IBR-CONICET-UNR, Argentina). Finally, I greatly acknowledge the 519 extremely helpful revisions from the Editor and two reviewers.

520

521

\section{References}

523

524

525

526

527

528

529

530

Abriata LA. 2017a. Web Apps Come of Age for Molecular Sciences. Informatics 4:28. DOI: 10.3390/informatics4030028.

Abriata LA. 2017b. Concurrent interactive visualization and handling of molecular structures over the Internet in web browsers. ArXiv e-prints.

Abriata LA, Palzkill T, Dal Peraro M. 2015. How structural and physicochemical determinants shape sequence constraints in a functional enzyme. PloS One 10:e0118684. DOI: 10.1371/journal.pone.0118684. 
531 Abriata LA, Rodrigues JPGLM, Salathé M, Patiny L. 2017. Augmenting research, education and 532 outreach with client-side web programming. Trends in Biotechnology.

533 Abriata LA, Tamò GE, Dal Peraro M. 2019. A further leap of improvement in tertiary structure 534 prediction in CASP13 prompts new routes for future assessments. Proteins. DOI:

$535 \quad$ 10.1002/prot.25787.

536 Abriata LA, Tamo G, Monastyrskyy M, Kryshtafovych A, Dal Peraro M. 2018. Assessment of 537 hard target modeling in CASP12 reveals an emerging role of alignment-based contact 538 prediction methods. Proteins: Structure, Function, and Bioinformatics 86 Suppl 1:97$539 \quad 112$.

540 Aspuru-Guzik A, Lindh R, Reiher M. 2018. The Matter Simulation (R)evolution. ACS Central $541 \quad$ Science 4:144-152. DOI: 10.1021/acscentsci.7b00550.

542 Ater T. 2019. Annyang. https://github.com/TalAter/annyang.

543 Bach B, Sicat R, Beyer J, Cordeil M, Pfister H. 2018. The Hologram in My Hand: How Effective 544 is Interactive Exploration of 3D Visualizations in Immersive Tangible Augmented

545

546 Reality? IEEE transactions on visualization and computer graphics 24:457-467. DOI:

547 Balo AR, Wang M, Ernst OP. 2017. Accessible virtual reality of biomolecular structural models 548 using the Autodesk Molecule Viewer. Nature Methods 14:1122-1123. DOI:

549 10.1038/nmeth.4506.

Bartók AP, De S, Poelking C, Bernstein N, Kermode JR, Csányi G, Ceriotti M. 2017. Machine learning unifies the modeling of materials and molecules. Science Advances 3. DOI: 10.1126/sciadv.1701816.

Berry C, Board J. 2014. A Protein in the palm of your hand through augmented reality. Biochemistry and Molecular Biology Education: A Bimonthly Publication of the International Union of Biochemistry and Molecular Biology 42:446-449. DOI: 10.1002/bmb.20805. 
557 Bertini I, Turano P, Vila AJ. 1993. NMR of paramagnetic metalloproteins. Chem.Rev. 93:28335582932.

559 Bolopion A, Cagneau B, Redon S, Régnier S. 2009. Haptic feedback for molecular simulation. $560 \quad$ DOI: 10.1109/IROS.2009.5354256.

561 Bolopion A, Cagneau B, Redon S, Régnier S. 2010. Comparing position and force control for 562 interactive molecular simulators with haptic feedback. Journal of Molecular Graphics \& $563 \quad$ Modelling 29:280-289. DOI: 10.1016/j.jmgm.2010.06.003.

564 Debye P. 1915. Zerstreuung von Röntgenstrahlen. Annalen der Physik 351:809-823. DOI: $565 \quad$ 10.1002/andp.19153510606.

566 Gillet A, Sanner M, Stoffler D, Goodsell D, Olson A. 2004. Augmented reality with tangible auto567 fabricated models for molecular biology applications.

568 Gillet A, Sanner M, Stoffler D, Olson A. 2005. Tangible interfaces for structural molecular 569 biology. Structure (London, England: 1993) 13:483-491. DOI: 10.1016/j.str.2005.01.009.

570 Goddard TD, Brilliant AA, Skillman TL, Vergenz S, Tyrwhitt-Drake J, Meng EC, Ferrin TE. 571 2018a. Molecular Visualization on the Holodeck. Journal of Molecular Biology. DOI:

572 10.1016/j.jmb.2018.06.040.

573 Goddard TD, Huang CC, Meng EC, Pettersen EF, Couch GS, Morris JH, Ferrin TE. 2018b. 574 UCSF ChimeraX: Meeting modern challenges in visualization and analysis. Protein 575 Science: A Publication of the Protein Society 27:14-25. DOI: 10.1002/pro.3235.

576 Hanson RM, Prilusky J, Renjian Z, Nakane T, Sussman JL. 2013. JSmol and the Next577 Generation Web-Based Representation of 3D Molecular Structure as Applied to Proteopedia. Israel Journal of Chemistry 53:207-216. DOI: 10.1002/ijch.201300024.

579 Hendler J. 2003. Science and the Semantic Web. Science 299:520. DOI: 10.1126/science.1078874. 
581 Hirano S, Kawasaki M, Ura H, Kato R, Raiborg C, Stenmark H, Wakatsuki S. 2006. Double582 sided ubiquitin binding of Hrs-UIM in endosomal protein sorting. Nature Structural \& $583 \quad$ Molecular Biology 13:272-277. DOI: 10.1038/nsmb1051.

584 Hirst JD, Glowacki DR, Baaden M. 2014. Molecular simulations and visualization: introduction 585 and overview. Faraday Discuss. 169:9-22. DOI: 10.1039/C4FD90024C.

586 Humphrey W, Dalke A, Schulten K. 1996. VMD - Visual Molecular Dynamics. Journal of $587 \quad$ Molecular Graphics 14:33-38.

588 J. Gimeno, S. Casas, C. Portalés, M. Fernádez. 2018. Addressing the Occlusion Problem in 589 Augmented Reality Environments with Phantom Hollow Objects. In: 2018 IEEE 590

591 International Symposium on Mixed and Augmented Reality Adjunct (ISMAR-Adjunct).

592 21-24. DOI: 10.1109/ISMAR-Adjunct.2018.00024.

Jiang C, Jin X. 2017. Quick Way to Port Existing C/C++ Chemoinformatics Toolkits to the Web 593 Using Emscripten. Journal of Chemical Information and Modeling. DOI:

594 10.1021/acs.jcim.7b00434.

Kato H. 1999. ARToolKit. http://www.hitl.washington.edu/artoolkit/.

Lee J, Kim J-I, Kang L-W. 2012. A Collaborative Molecular Modeling Environment Using a Virtual Tunneling Service. Journal of Biomedicine and Biotechnology 2012:7.

M. DiPierro. 2018. The Rise of JavaScript. Computing in Science \& Engineering 20:9-10. DOI: 10.1109/MCSE.2018.011111120.

M. Fjeld, B. M. Voegtli. 2002. Augmented Chemistry: an interactive educational workbench. In: Proceedings. International Symposium on Mixed and Augmented Reality. 259-321. DOI: 10.1109/ISMAR.2002.1115100.

M. Krichenbauer, G. Yamamoto, T. Taketom, C. Sandor, H. Kato. 2018. Augmented Reality 604 605 versus Virtual Reality for 3D Object Manipulation. IEEE Transactions on Visualization and Computer Graphics 24:1038-1048. DOI: 10.1109/TVCG.2017.2658570. 
606 M. M. Shah, H. Arshad, R. Sulaiman. 2012. Occlusion in augmented reality. In: 2012 8th

607

608

609

610

611

612

613

614

615

616

617

618

619

620

621

622

623

624

625

626

627

628

629

630

631

International Conference on Information Science and Digital Content Technology (ICIDT2012). 372-378.

Maier P, Klinker G. 2013. Augmented Chemical Reactions: 3D Interaction Methods for Chemistry. International Journal of Online Engineering 9.

Maier P, Tönnis M, GudrunKlinker D. 2009. Dynamics in Tangible Chemical Reactions. In: World Academy of Science, Engineering and Technology.

Marko JF, Siggia ED. 1995. Statistical mechanics of supercoiled DNA. Phys. Rev. E 52:29122938. DOI: 10.1103/PhysRevE.52.2912.

Marrink SJ, Risselada HJ, Yefimov S, Tieleman DP, de Vries AH. 2007. The MARTINI force field: coarse grained model for biomolecular simulations. The Journal of Physical Chemistry. B 111:7812-7824. DOI: 10.1021/jp071097f.

Martínez-Hung H, García-López CA, Escalona-Arranz JC. 2016. Augmented Reality Models Applied to the Chemistry Education on the University (article in Spanish). Revista Cubana de Química 29:13-25.

Matthews D. 2018. Virtual-reality applications give science a new dimension. Nature 557:127128. DOI: $10.1038 / \mathrm{d} 41586-018-04997-2$.

Matthews N, Kitao A, Laycock S, Hayward S. 2019. Haptic-Assisted Interactive Molecular Docking Incorporating Receptor Flexibility. Journal of Chemical Information and Modeling 59:2900-2912. DOI: 10.1021/acs.jcim.9b00112.

Moore EB, Chamberlain JM, Parson R, Perkins KK. 2014. PhET Interactive Simulations: Transformative Tools for Teaching Chemistry. Journal of Chemical Education 91:11911197. DOI: $10.1021 /$ ed4005084.

Müller C, Krone M, Huber M, Biener V, Herr D, Koch S, Reina G, Weiskopf D, Ertl T. 2018. Interactive Molecular Graphics for Augmented Reality Using HoloLens. Journal of Integrative Bioinformatics 15. DOI: 10.1515/jib-2018-0005.

Peer] Comput. Sci. reviewing PDF | (CS-2019:03:36241:3:0:NEW 9 Jan 2020) 
632 O'Connor M, Deeks HM, Dawn E, Metatla O, Roudaut A, Sutton M, Thomas LM, Glowacki BR, 633 Sage R, Tew P, Wonnacott M, Bates P, Mulholland AJ, Glowacki DR. 2018. Sampling 634 molecular conformations and dynamics in a multiuser virtual reality framework. Science $635 \quad$ Advances 4:eaat2731. DOI: 10.1126/sciadv.aat2731.

636 O'Donoghue SI, Gavin A-C, Gehlenborg N, Goodsell DS, Hériché J-K, Nielsen CB, North C, 637 Olson AJ, Procter JB, Shattuck DW, Walter T, Wong B. 2010. Visualizing biological data638 now and in the future. Nature Methods 7:S2-4. DOI: 10.1038/nmeth.f.301.

639 Ovchinnikov S, Kamisetty H, Baker D. 2014. Robust and accurate prediction of residue-residue 640 interactions across protein interfaces using evolutionary information. eLife 3:e02030. DOI: 10.7554/eLife.02030.

642 Pafilis E, O’Donoghue SI, Jensen LJ, Horn H, Kuhn M, Brown NP, Schneider R. 2009. Reflect:

643 augmented browsing for the life scientist. Nature Biotechnology 27:508-510. DOI:

644 10.1038/nbt0609-508.

Papoutsaki A, Sangkloy P, Laskey J, Daskalova N, Huang J, Hays J. 2016. WebGazer: Scalable Webcam Eye Tracking Using User Interactions. In: Proceedings of the 25th

647 International Joint Conference on Artificial Intelligence. 3839-3845.

648

Paruzzo FM, Hofstetter A, Musil F, De S, Ceriotti M, Emsley L. Chemical Shifts in Molecular 649 Solids by Machine Learning. ArXiv 1805:11541.

Pence HE, Williams AJ, Belford RE. 2015. New Tools and Challenges for Chemical Education: Mobile Learning, Augmented Reality, and Distributed Cognition in the Dawn of the Social and Semantic Web. In: Chemistry Education. Wiley-VCH Verlag GmbH \& Co. KGaA, 693-734. DOI: 10.1002/9783527679300.ch28.

Q. H. Gao, T. R. Wan, W. Tang, L. Chen. 2017. A Stable and Accurate Marker-Less Augmented 656 Reality Registration Method. In: 2017 International Conference on Cyberworlds (CW). 41-47. DOI: 10.1109/CW.2017.44. 
657 Ratamero EM, Bellini D, Dowson CG, Römer RA. 2018. Touching proteins with virtual bare 658 hands : Visualizing protein-drug complexes and their dynamics in self-made virtual reality using gaming hardware. Journal of Computer-Aided Molecular Design 32:703709. DOI: $10.1007 / \mathrm{s} 10822-018-0123-0$.

Rebholz-Schuhmann D, Oellrich A, Hoehndorf R. 2012. Text-mining solutions for biomedical research: enabling integrative biology. Nature Reviews. Genetics 13:829-839. DOI:

Rego N, Koes D. 2015. 3Dmol.js: molecular visualization with WebGL. Bioinformatics (Oxford, England) 31:1322-1324. DOI: 10.1093/bioinformatics/btu829.

Rose AS, Hildebrand PW. 2015. NGL Viewer: a web application for molecular visualization. Nucleic Acids Research 43:W576-579. DOI: 10.1093/nar/gkv402.

Russel D, Lasker K, Webb B, Velázquez-Muriel J, Tjioe E, Schneidman-Duhovny D, Peterson B, Sali A. 2012. Putting the pieces together: integrative modeling platform software for

670

671 structure determination of macromolecular assemblies. PLoS biology 10:e1001244. DOI: 10.1371/journal.pbio.1001244.

S. Doutreligne, T. Cragnolini, S. Pasquali, P. Derreumaux, M. Baaden. 2014. UnityMol: Interactive scientific visualization for integrative biology. In: 2014 IEEE 4th Symposium on Large Data Analysis and Visualization (LDAV). 109-110. DOI: S, Koča J. 2017. LiteMol suite: interactive web-based visualization of large-scale macromolecular structure data. Nature Methods in press.

679 Sehnal D, Rose AS, Koča J, Burley SK, Velankar S. 2018. Mol*: Towards a Common Library and Tools for Web Molecular Graphics. In: Proceedings of the Workshop on Molecular Graphics and Visual Analysis of Molecular Data. MolVA '18. Goslar Germany, Germany: Eurographics Association, 29-33. 
683 Sekhavat YA, Zarei H. 2016. Enhancing the Sense of Immersion and Quality of Experience in 684 Mobile Games Using Augmented Reality. Journal of Computing and Security 3:53-62. 685 Simkovic F, Ovchinnikov S, Baker D, Rigden DJ. 2017. Applications of contact predictions to 686 structural biology. IUCrJ 4:291-300. DOI: 10.1107/S2052252517005115.

687 Smith JS, Isayev O, Roitberg AE. 2017. ANI-1: an extensible neural network potential with DFT 688 accuracy at force field computational cost. Chemical Science 8:3192-3203. DOI: $689 \quad 10.1039 / c 6 s c 05720 a$.

690 Sommer B, Baaden M, Krone M, Woods A. 2018. From Virtual Reality to Immersive Analytics in 691 Bioinformatics. Journal of Integrative Bioinformatics 15. DOI: 10.1515/jib-2018-0043.

692 Stocks MB, Hayward S, Laycock SD. 2009. Interacting with the biomolecular solvent accessible 693 surface via a haptic feedback device. BMC structural biology 9:69. DOI: 10.1186/1472-

694 6807-9-69.

695

Stocks MB, Laycock SD, Hayward S. 2011. Applying forces to elastic network models of large 696 biomolecules using a haptic feedback device. Journal of Computer-Aided Molecular

697 Design 25:203-211. DOI: 10.1007/s10822-010-9410-0.

698

Stone J. 2019. VMD support for VR and Interactive MD.

699 https://www.ks.uiuc.edu/Research/vmd/allversions/interactive_MD.html:

700 https://www.ks.uiuc.edu/Research/vmd/allversions/interactive_MD.html.

701 Stovgaard K, Andreetta C, Ferkinghoff-Borg J, Hamelryck T. 2010. Calculation of accurate small 702 703 angle X-ray scattering curves from coarse-grained protein models. BMC bioinformatics 11:429. DOI: 10.1186/1471-2105-11-429.

V. Pimentel, B. G. Nickerson. 2012. Communicating and Displaying Real-Time Data with WebSocket. IEEE Internet Computing 16:45-53. DOI: 10.1109/MIC.2012.64.

Vega Garzón JC, Magrini ML, Galembeck E. 2017. Using augmented reality to teach and learn biochemistry. Biochemistry and Molecular Biology Education: A Bimonthly Publication of 
708

709

\section{0}

711

712

713

714

715

716

717

718

719

720

721

722

723

724

725

726

727

728

729

the International Union of Biochemistry and Molecular Biology. DOI: 10.1002/bmb.21063.

Wiebrands M, Malajczuk CJ, Woods AJ, Rohl AL, Mancera RL. 2018. Molecular Dynamics Visualization (MDV): Stereoscopic 3D Display of Biomolecular Structure and Interactions Using the Unity Game Engine. Journal of Integrative Bioinformatics 15. DOI: 10.1515/jib2018-0010.

Wollacott AM, Merz KM. 2007. Haptic applications for molecular structure manipulation. Journal of Molecular Graphics \& Modelling 25:801-805. DOI: 10.1016/j.jmgm.2006.07.005.

Wolle P, Müller MP, Rauh D. 2018. Augmented Reality in Scientific Publications-Taking the Visualization of 3D Structures to the Next Level. ACS Chemical Biology 13:496-499. DOI: 10.1021/acschembio.8b00153.

Xu K, Liu N, Xu J, Guo C, Zhao L, Wang H-W, Zhang QC. 2019. VRmol: an Integrative CloudBased Virtual Reality System to Explore Macromolecular Structure. bioRxiv:589366. DOI: $10.1101 / 589366$.

Y. Gaffary, B. Le Gouis, M. Marchal, F. Argelaguet, B. Arnaldi, A. Lécuyer. 2017. AR Feels "Softer" than VR: Haptic Perception of Stiffness in Augmented versus Virtual Reality. IEEE Transactions on Visualization and Computer Graphics 23:2372-2377. DOI: 10.1109/TVCG.2017.2735078.

Yang S, Mei B, Yue X. 2018. Mobile Augmented Reality Assisted Chemical Education: Insights from Elements 4D. Journal of Chemical Education 95:1060-1062. DOI: 10.1021/acs.jchemed.8b00017. 


\section{Figure 1}

Commodity augmented reality in web browsers

(A) AR markers "Hiro" and "Kanji" are built into AR.js and its A-Frame wrap. Figure S1 shows them ready to print at various sizes. Custom markers including cube markers are also implementable. (B) In the proposed web apps the AR library feeds coordinate information to the graphics libraries and other algorithms. (C) The web pages must be hosted in servers compatible with the https protocol, but their content then runs exclusively on the computer. 
A
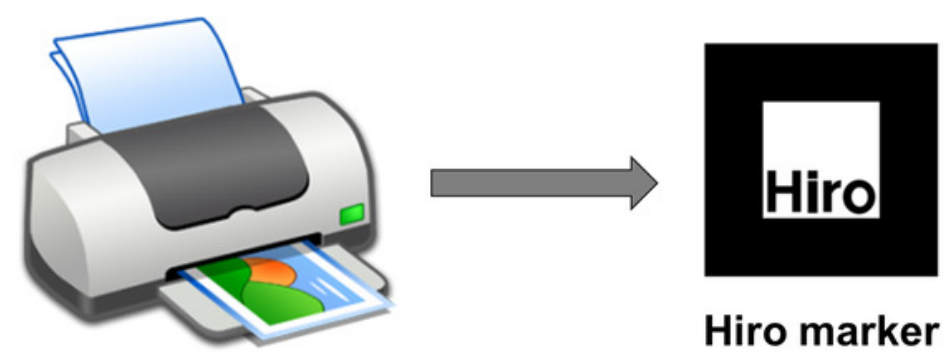

Hiro marker

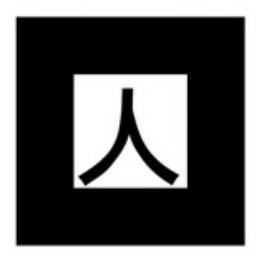

Kanji marker

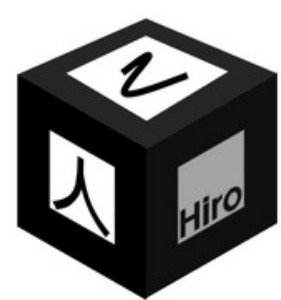

Cube marker
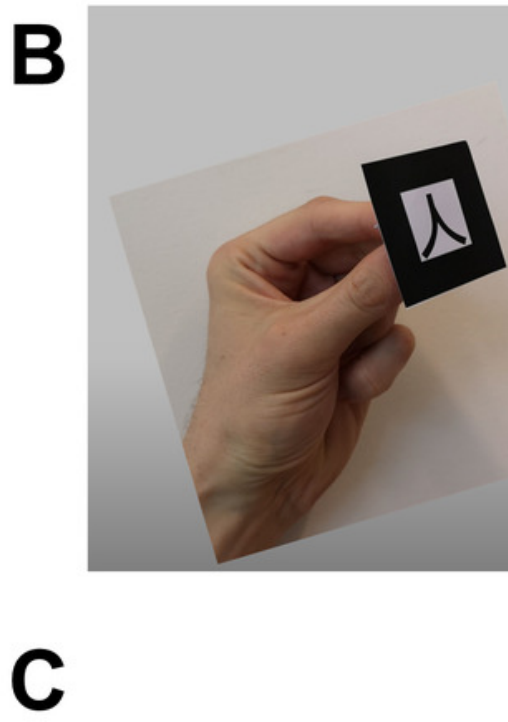

C

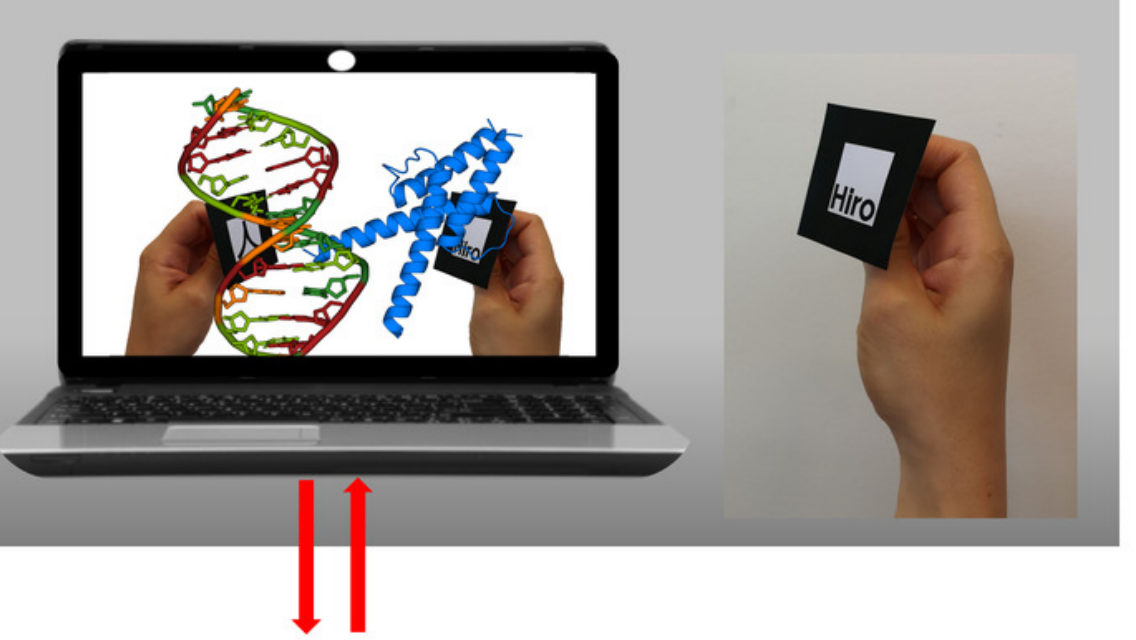

HTML CSS JS

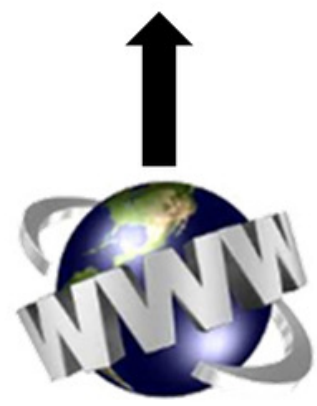




\section{Figure 2}

Different implementations of WebGL for AR in web browsers.

(A) 2-bromo-butane enantiomers $\mathrm{R}$ and $\mathrm{S}$ displayed on Hiro and Kanji markers, respectively, from Wavefront objects produced in VMD. (B) A protein complex rendered as cartoons with small molecule ligands shown as sticks on the Hiro marker (PDB ID IVYQ). (C) A doublestranded segment of DNA (sticks) bound to a homeodomain protein (cartoon) spinning on the Kanji marker (PDB ID 1FJL). Both B and C were rendered from VMD Wavefront objects. (D) Volumetric map of a bacteriophage attached to a portion of the host's cell wall as determined through electron microscopy (EMDB ID 9010) and prepared as VMD Wavefront object showing the capsid in grey, the needle in blue and the membranes in orange. (E) A small peptide inside its electron density map as determined by $x$-ray diffraction (PDB ID 3HYD). (F) HOMO and LUMO orbitals from $\mathrm{NH}_{3}$ and $\mathrm{BH}_{3}$ molecules, rendered from Wavefront objects. (G) Representation of an amphipathic alpha helix built from primitives, viewable on the Kanji marker. (H) Use of a cube marker (made up of 6 different AR markers in its faces) to load any molecule in PDB format and handle and visualize it in 3D. Graphics built from Three.js primitives. The example also uses Cannon.js to simulate rigid body dynamics by fixing the distances between atoms separated by 1 or 2 bonds but allowing rotations, in analogy to plastic-made molecular modeling kits. 

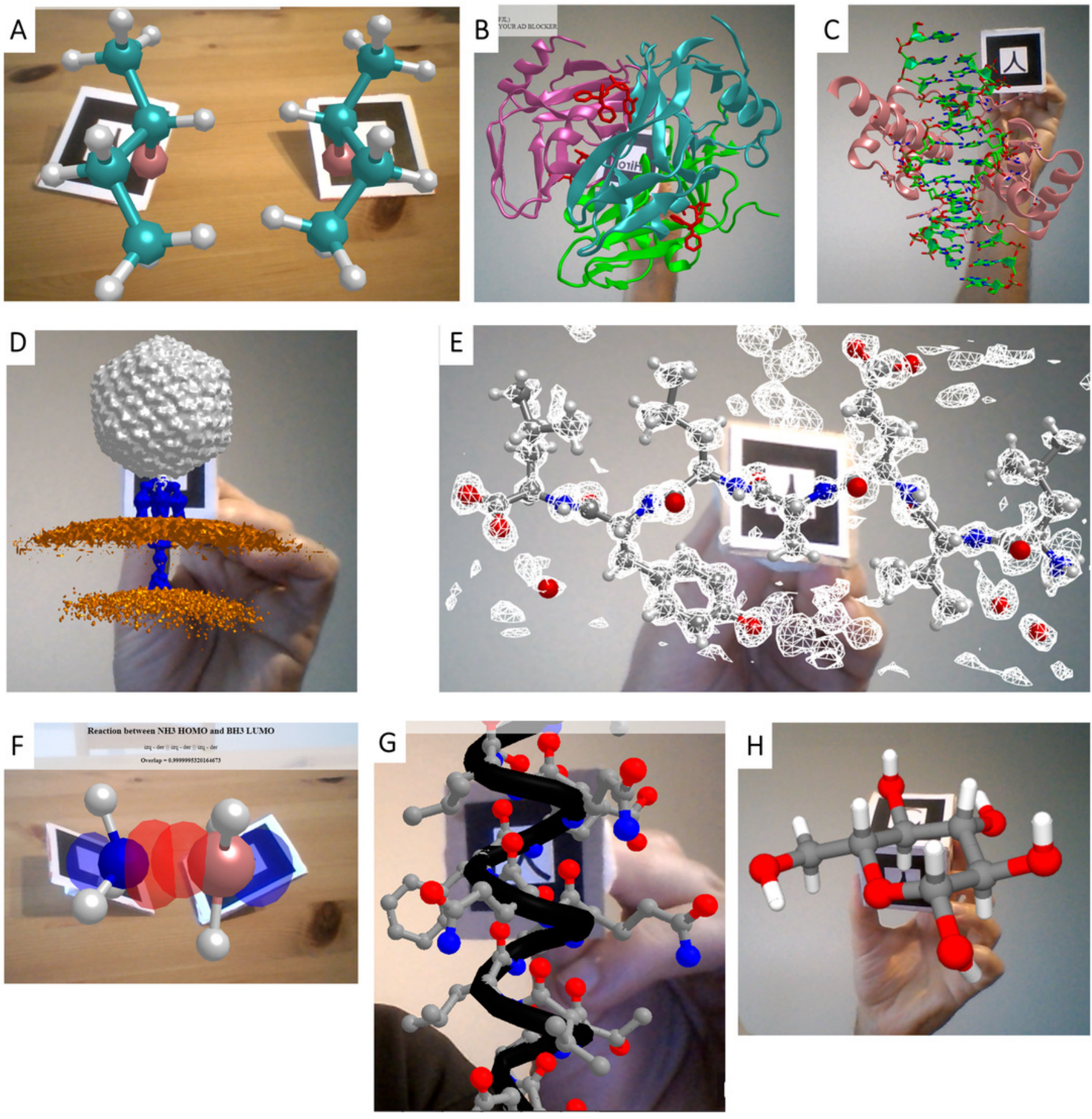


\section{Figure 3}

Introducing interactivity

(A) A lysine and a glutamate side chains attached to different AR markers, whose coordinates are processed in real time to deliver distance and electrostatic potential between charged groups and to calculate and display clashes. Graphics achieved with A-Frame polyfills. In this example the ratio of protonated lysine to glutamate exchanging dynamically is set to $70 / 30$ $=2.33$, i.e. favoring protonated lysine as the actual water chemistry dictates but shifted orders of magnitude from the real ratio of acidic constants so that the user can observe temporal protonation of both amino acids in the timescale of work. (B) A Diels-Alder reaction, taking place once the user has moved the reagents close enough in space; this example further helps to visualize fused cycles (as the diene reagent was a cyclic molecule itself). (C) Example of interactively probing experimental observables: as a probe atom (black sphere) is moved around a paramagnetic center, the user sees the paramagnetic effects simulated at the location of the probe atom in real time. 

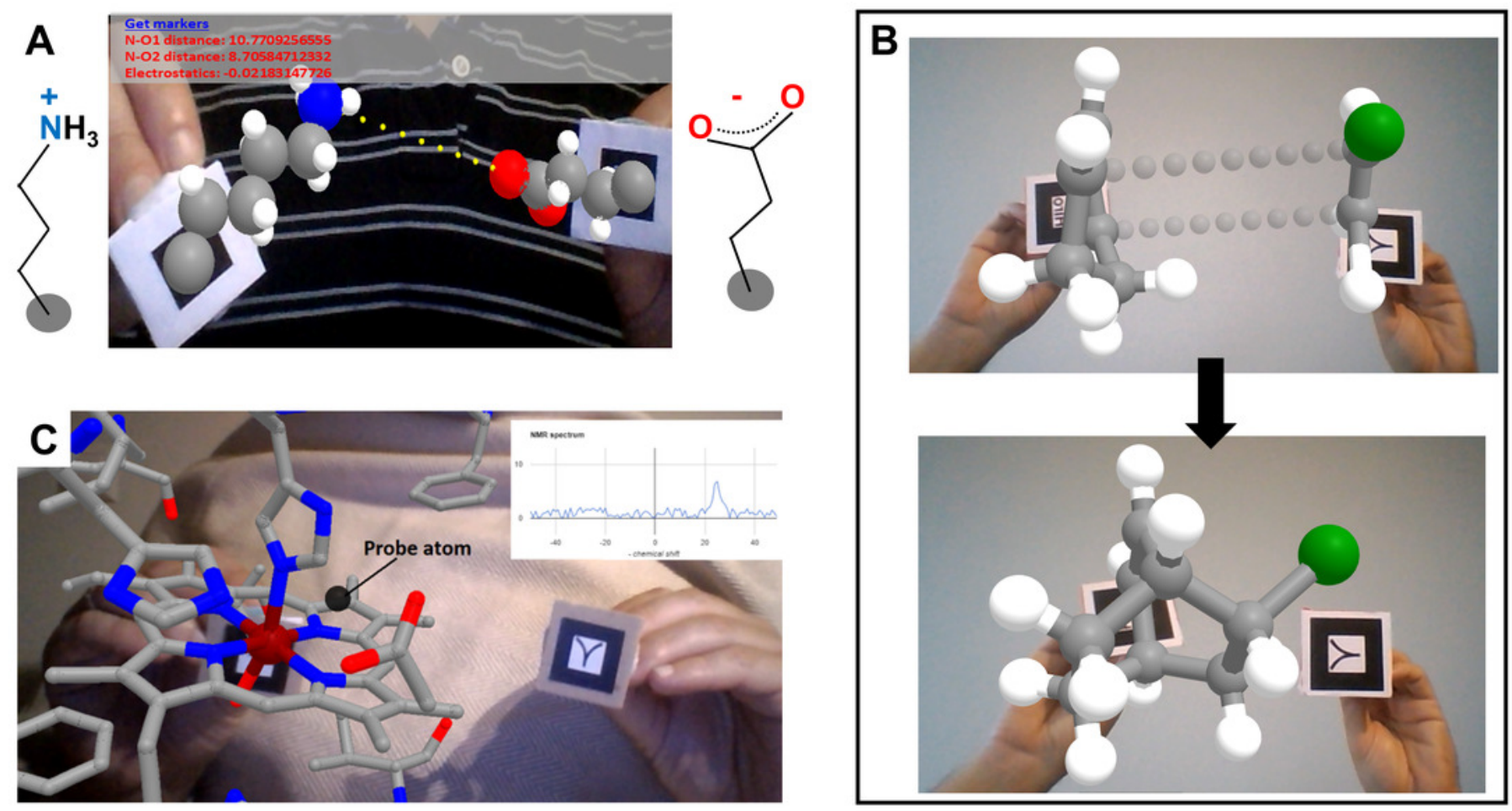


\section{Figure 4}

Interactivity in biological macromolecules

(A) Ubiquitin and ubiquitin-interacting motif (red and blue respectively, taken from PDB ID 2D3G) driven in 3D with two AR markers, as the web app computes the predicted SAXS profile and displays it overlaid on top of a purported experimental profile together with a metric of the fit quality. (B) Interactive exploration of contacts predicted between two proteins (here from coevolution data) before and after manual docking. This example is 1QOP from Ovchinnikov et al, where contacts predicted with high score are colored green, contacts of intermediate confidence are olive, and the first contact of low probability is shown red (as taken from the original data). The thickness of the contact lines indicates distance, such that thin lines indicate the residues are close in space. Notice how the red contact, which has low probability, remains thicker than the well-scored contacts (green) upon manual docking.

A
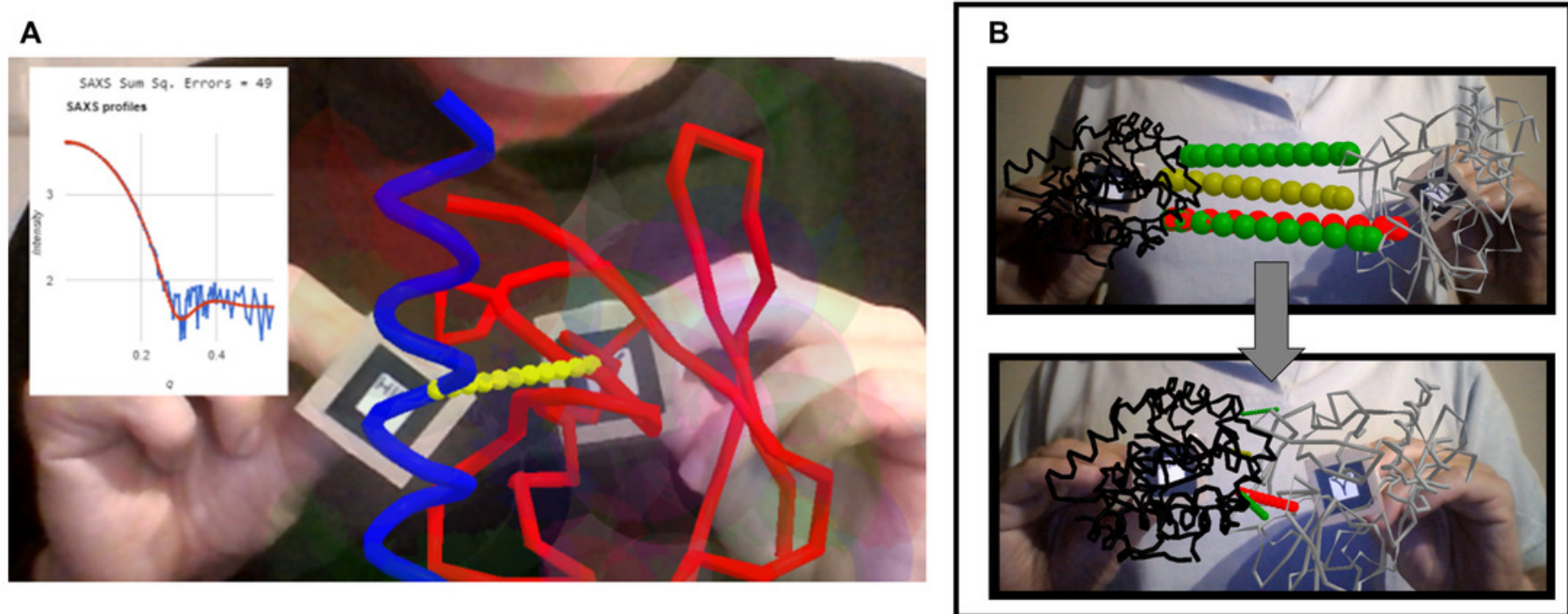


\section{Figure 5}

Dynamics of highly disordered protein linkers modeled with rigid-body mechanics

Ubiquitin and ubiquitin-interacting motif (PDB ID 2D3G) modelled as 2-4 beads per residue, colored by physicochemical properties (grey=hydrophobic, red=negative, blue=positive, green=polar uncharged; backbone beads in black). The domains are driven independently in 3D with two AR markers. They are connected through a flexible linker of six backbone-sized beads (orange) whose dynamics are treated with the Cannon.js rigid-body force field. The web app reports in real time the distance between the centers of both domains, the entropy of the linker based on a worm-like chain model, and the linker strain computed from deviations of distances between consecutive linker beads from an equilibrium distance. (A) The two domains extended as much as possible while keeping the linker relaxed (although entropically unfavoured) illustrates the maximum possible separation with the given linker is of around $40 \AA$. (B) The binding pose between the 2 domains is geometrically feasible as it keeps the linker relaxed. (C) This other binding pose is unachievable with a linker of this length. 


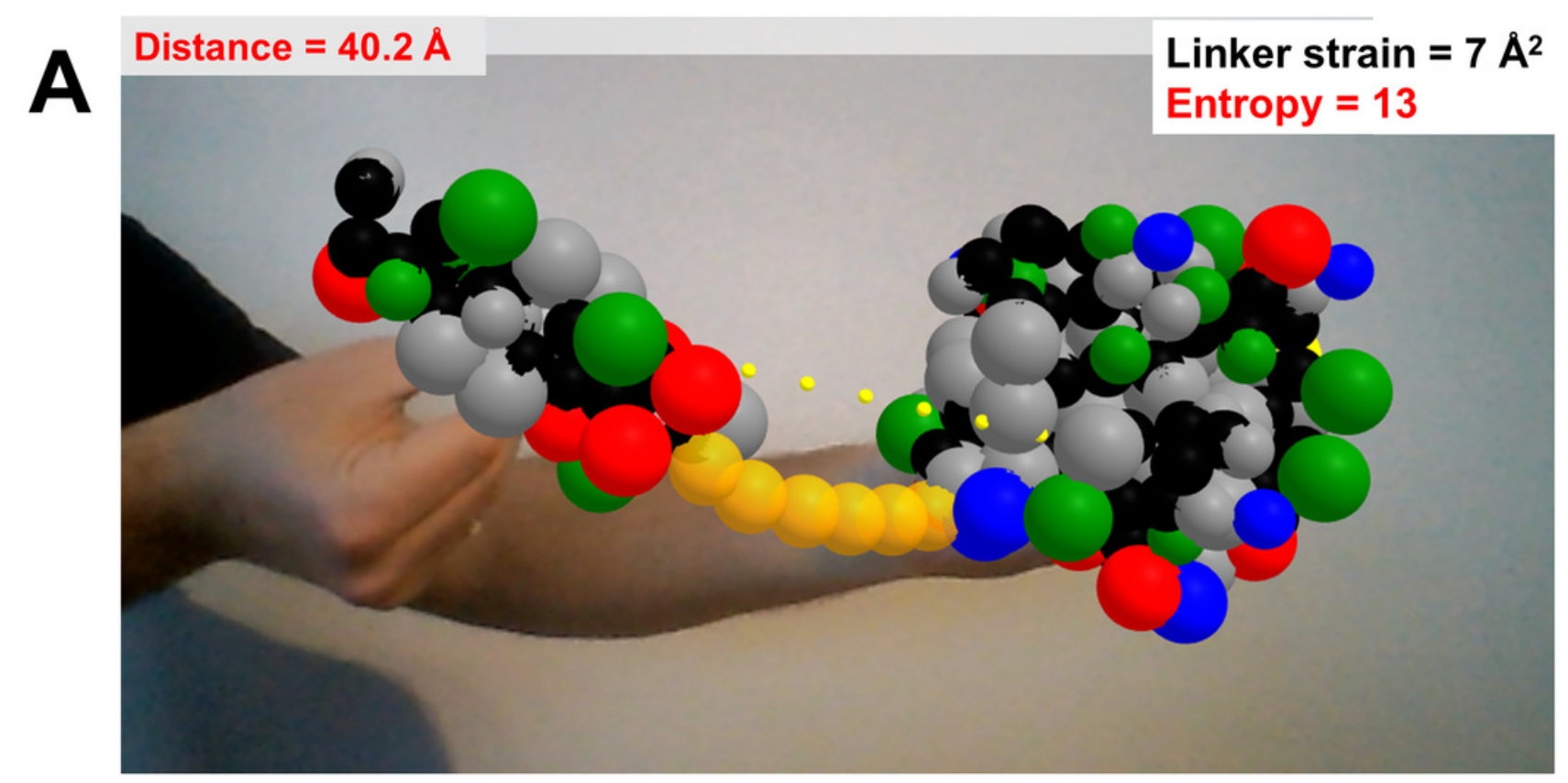

B Distance $=17.7 \AA$

Linker strain $=2 \AA^{2}$

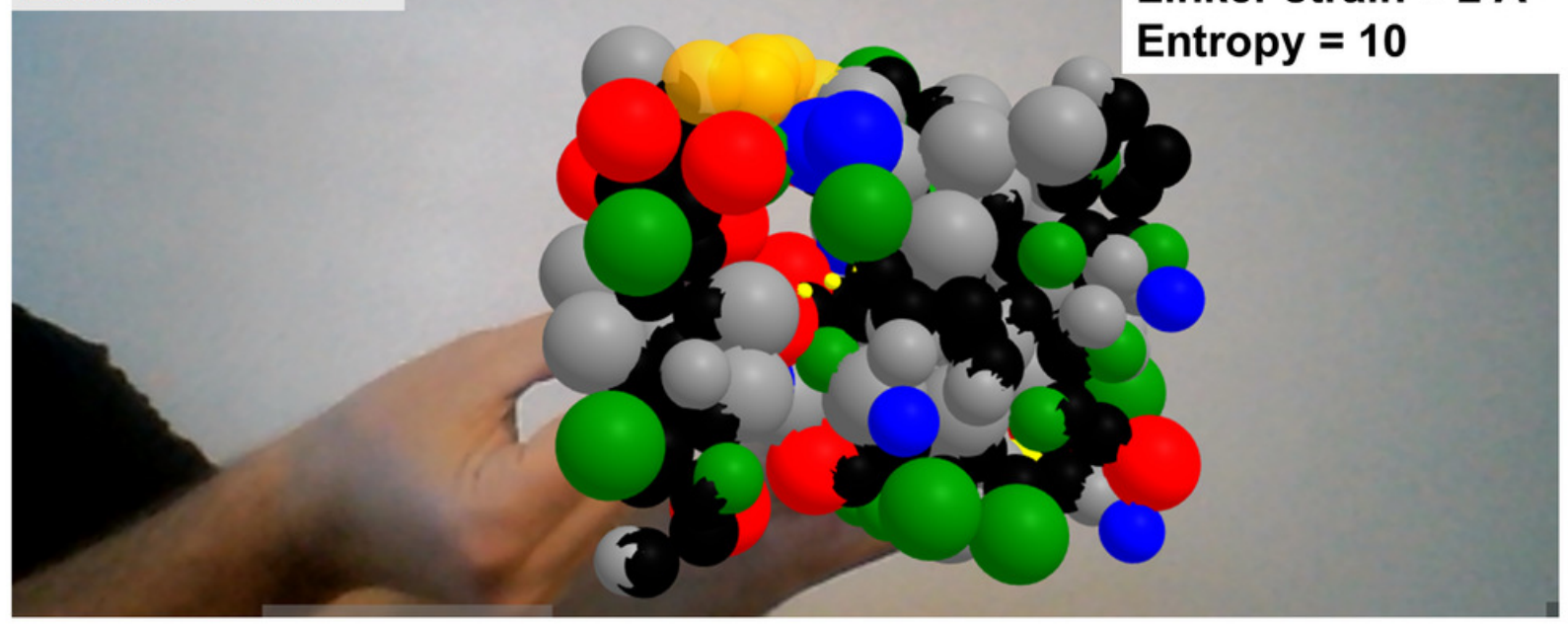

C Distance $=26.2 \AA$

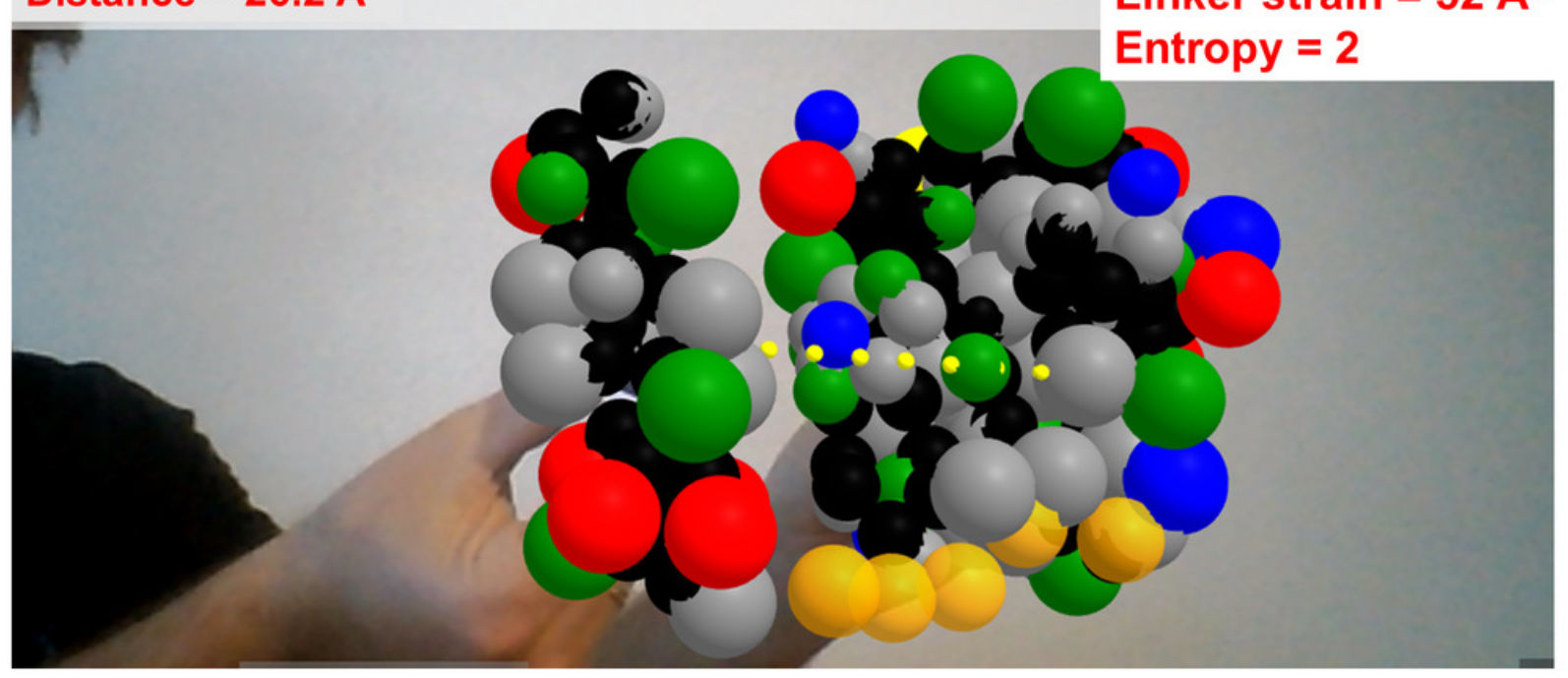




\section{Table $\mathbf{1}$ (on next page)}

Links to all web examples arranged by figure

See https://lucianoabriata.altervista.org/papersdata/tablepeerjcs2019.html for an online version of the table which also includes links to several video demonstrations. 
1 Table 1. Links to all web examples arranged by figure. See

2 https://lucianoabriata.altervista.org/papersdata/tablepeerjcs2019.html for an online version of the table

3 which also includes links to several video demonstrations.

4

Figure 2

2A https://lucianoabriata.altervista.org/isinscience/aris/armodeling/2brbutane.html

2B,C https://lucianoabriata.altervista.org/jsinscience/arjs/armodeling/pdb-1vyq-1fjl.html

2D https://lucianoabriata.altervista.org/jsinscience/aris/armodeling/bacteriophage.html

$2 \mathrm{E} \quad$ https://lucianoabriata.altervista.org/isinscience/arjs/armodeling/xray3hyd.html

2F https://lucianoabriata.altervista.org/isinscience/arjs/armodeling/bh3nh3orb.html

$2 \mathrm{G}$ https://lucianoabriata.altervista.org/isinscience/arjs/armodeling/ubiquitinandNESatomistic.html

$2 \mathrm{H} \quad \underline{\text { https://lucianoabriata.altervista.org/isinscience/arjs/isartoolkit5/pdbloader6.html }}$

Figure 3

3A https://lucianoabriata.altervista.org/jsinscience/arjs/armodeling/smallmolclashdetection.html and https://lucianoabriata.altervista.org/jsinscience/aris/armodeling/smallmolprotontransfer.html

3B https://lucianoabriata.altervista.org/isinscience/arjs/armodeling/smallmoldielsalder.html

3C https://lucianoabriata.altervista.org/jsinscience/arjs/armodeling/metmyoglobinfe3pcshift.html

\section{Figure 4}

4A https://lucianoabriata.altervista.org/isinscience/arjs/armodeling/ubiquitinuimffvoicesaxs.html

4B-C https://lucianoabriata.altervista.org/jsinscience/arjs/armodeling/coevol 1qop.html

Figure 5

5A-C https://lucianoabriata.altervista.org/isinscience/arjs/armodeling/ubiquitin-uim-cannon.html

5

6 


\section{Table 2 (on next page)}

Requirements and troubleshooting for developers and users 
1 Table 2. Requirements and troubleshooting for developers and users.

Developers

Software and hardware requirements

* Ensure using https URLs; otherwise webcams will not activate.

* Free web hosting services work, as web pages only need to be hosted but run in the client.

* Given the regular updates in w3c standards, APIs and libraries, routine tests are recommended to ensure normal functioning.

* Examples from this paper were verified to work "out of the box" on Safari in multiple MacOS 10.x versions and on multiple Chrome and Firefox versions in Windows 8, Windows 10, Linux RedHat Enterprise Edition, Ubuntu and ArchLinux.

* Currently limited and heterogeneous support in tablets and smartphones, these devices are not recommended.

Users

Software and hardware requirements

* Need a webcam- and internet-enabled computer (desktop or laptop).

* Enable webcam when prompted.

* JavaScript and WebGL must be enabled in the browser (that is a default setting).

* Check that ad blockers and firewalls do not block the webcam and other content.

* Pages containing large Wavefront files may take time to load (half to a few minutes).

Augmented reality markers

* Print on regular printer; try different sizes for different applications.

* When using Hiro and Kanji markers ensure they are printed at the same size.

* Ensure that makers have a white frame around the black drawing, at least $10 \%$ of size.

* To improve marker recognition, avoid glossy papers. Opaque paper is best.

* Lighting may also affect the quality of the AR experience.

* Markers are easier to handle if glued on solid surfaces (but avoid wrinkles).

* Cubic marker can be glued on solid rubber cube cut to appropriate size. 